\title{
Convergence analysis of a Halpern-like iterative algorithm in Hilbert spaces
}

\author{
Yunpeng Zhang ${ }^{\mathrm{a}}$, Sun Young Cho ${ }^{\mathrm{b}, *}$ \\ ${ }^{a}$ College of Electric Power, North China University of Water Resources and Electric Power, Zhengzhou 450011, China. \\ ${ }^{b}$ Center for General Education, China Medical University, Taichung 40402, Taiwan.
}

Communicated by Y. Z. Chen

\begin{abstract}
In this paper, a Halpern-like iterative algorithm is investigated for finding a solution of a split feasibility problem and a solution to a nonexpansive operator equation. Strong convergence theorems are established in the framework of infinite dimensional Hilbert spaces. (C)2017 All rights reserved.
\end{abstract}

Keywords: Convergence analysis, Hilbert space, monotone mapping, split feasibility problem. 2010 MSC: 47H06, 90C33.

\section{Introduction and preliminaries}

Let $\mathrm{C}$ and $\mathrm{Q}$ be nonempty closed and convex subsets of real Hilbert spaces $\mathrm{H}_{1}$ and $\mathrm{H}_{2}$, respectively, and let $\operatorname{Proj}_{C}$ and $\operatorname{Proj}_{Q}$ be the metric projections from $H_{1}$ and $H_{2}$ to $C$ and $Q$, respectively, and $A: H_{1} \rightarrow H_{2}$ be a bounded linear operator.

Recall that the split feasibility problem is formulated as to find a point $\mathrm{q} \in \mathrm{H}_{1}$ such that:

$$
\mathrm{q} \in \mathrm{C} \text { and } \mathrm{Aq} \in \mathrm{Q} \text {. }
$$

It is easy to see that $q \in \mathrm{H}_{1}$ solves equation (1.1) if and only if it solves the following fixed point equation

$$
q=\operatorname{Proj}_{C}\left(I-\gamma A^{*}\left(I-P_{Q}\right) A\right) q, \quad x \in C,
$$

where $A^{*}$ is the adjoint of $A$.

In 1994, Censor and Elfving [6] introduced the split feasibility problem in finite dimensional Hilbert spaces for modeling inverse problems which arise from phase retrievals and in medical image reconstruction. It has been found that the split feasibility problem can also be used in various disciplines such as image restoration, computer tomograph and radiation therapy treatment planning $[4,5,7]$.

Recently, Byrne [4] considered the split feasibility problem in an infinite dimensional Hilbert space. In many disciplines, including image restoration, computer tomograph, control theory, and quantum

\footnotetext{
*Corresponding author

Email addresses: zhangypliyl@yeah.net (Yunpeng Zhang), ooly61@hotmail.com (Sun Young Cho)
} 
physics, problems arise in infinite dimension spaces. Therefore, it is important to consider the split feasibility problem in the framework of infinite dimensional spaces. On the other hand, the split feasibility problem covers convex feasibility problems that it is to find a common element in the intersection of a family of closed and convex subsets of Hilbert spaces. Recently, many authors have studied the split feasibility problem via fixed point methods; see $[8,11,15,17-19]$ and the references therein.

Let $\mathrm{H}$ be a Hilbert space. Recall that a mapping $\mathrm{T}: \mathrm{H} \rightarrow \mathrm{H}$ is said to be monotone iff

$$
\langle T x-T y, x-y\rangle \geqslant 0, \quad \forall x, y \in H .
$$

$\mathrm{T}: \mathrm{H} \rightarrow \mathrm{H}$ is said to be inverse-strongly monotone iff there exists a constant $v>0$ such that

$$
\langle T x-T y, x-y\rangle \geqslant v\|T x-T y\|^{2}, \quad \forall x, y \in H .
$$

We also say that $\mathrm{T}$ is $\mathrm{v}$-inverse-strongly monotone. It is obvious that if $\mathrm{F}$ is $v$-inverse-strongly monotone, then it is $\frac{1}{v}$-Lipschitz continuous and monotone.

Recall that a mapping $\mathrm{T}: \mathrm{H} \rightarrow \mathrm{H}$ is said to be nonexpansive iff

$$
\|\mathrm{T} x-\mathrm{T} y\| \leqslant\|x-y\|, \quad \forall x, y \in H .
$$

From Browder [3], we see that every nonexpansive mapping on bounded closed and convex subsets of Hilbert spaces has a nonempty fixed point set; see [3] and the references therein. Halpern-like iterative algorithms have recently investigated to study fixed points of nonexpansive mappings and zero points of monotone operators; see $[1,2,9,10,13,14,16]$ and the references therein. The advantage of Halpern-like iterative algorithms is that strong convergence can be guaranteed without any compact assumptions.

Let $\mathrm{D}$ be a nonempty closed and convex subset of real Hilbert space $\mathrm{H}$.

Recall that the metric projection $\mathrm{P}_{\mathrm{D}}^{\mathrm{H}}: \mathrm{H} \rightarrow \mathrm{D}$ from $\mathrm{H}$ onto $\mathrm{D}$ of $\mathrm{H}$ is defined as follows: for each point $x \in \mathrm{H}$, there exists a unique point $\mathrm{P}_{\mathrm{D}}^{\mathrm{H}} \mathrm{x} \in \mathrm{D}$ with the property:

$$
\left\|x-P_{D}^{\mathrm{H}} x\right\| \leqslant\|x-y\| .
$$

Thus for any $x \in H, \tilde{x}=P_{D}^{H} x$ iff $\tilde{x} \in D$ and $\|x-\tilde{x}\|=\inf \{\|x-y\|: y \in D\}$. We also have the following facts

$$
\begin{aligned}
\|x-y\|^{2}-\left\|\left(I-P_{D}^{H}\right) x-\left(I-P_{D}^{H}\right) y\right\|^{2} & \geqslant\left\|P_{D}^{H} x-P_{D}^{H} y\right\|^{2}, \quad \forall x, y \in H, \\
\left\langle\left(I-P_{D}^{H}\right) x-\left(I-P_{D}^{H}\right) y, x-y\right\rangle & \geqslant\left\|\left(I-P_{D}^{H}\right) x-\left(I-P_{D}^{H}\right) y\right\|^{2}, \quad \forall x, y \in H,
\end{aligned}
$$

and

$$
\left\langle P_{D}^{\mathrm{H}} x-P_{\mathrm{D}}^{\mathrm{H}} y, x-y\right\rangle \geqslant\left\|P_{\mathrm{D}}^{\mathrm{H}} x-\mathrm{P}_{\mathrm{D}}^{\mathrm{H}} y\right\|^{2}, \quad \forall x, y \in H .
$$

Lemma 1.1 ([12]). Let $\left\{a_{n}\right\}$ be a sequence of nonnegative real numbers such that

$$
a_{n+1} \leqslant\left(1-t_{n}\right) a_{n}+b_{n}, \quad \forall n \geqslant 0,
$$

where $\left\{t_{n}\right\} \subset(0,1)$ and $\left\{b_{n}\right\}$ is a sequence of real numbers. Assume that

$$
\sum_{n=0}^{\infty} t_{n}=\infty \text { and } \limsup _{n \rightarrow \infty} \frac{b_{n}}{t_{n}} \leqslant 0 .
$$

Then $\lim _{n \rightarrow \infty} a_{\mathfrak{n}}=0$.

Lemma 1.2 ([3]). Let $\mathrm{H}$ be a Hilbert space and let $\mathrm{S}$ be a nonexpansive mapping on $\mathrm{H}$. Then $\mathrm{I}-\mathrm{S}$ is demiclosed at the origin. That is, $\left\{x_{n}\right\}$ converges weakly to $p$ and $\left\{x_{n}-S x_{n}\right\}$ converges strongly to 0 . Then $p$ is a fixed point of $S$.

\section{Main results}

Theorem 2.1. Let $\mathrm{C}$ be a nonempty closed and convex subset of Hilbert space $\mathrm{H}_{1}$ and let $\mathrm{Q}$ be a nonempty closed and convex subset of Hilbert space $\mathrm{H}_{2}$. Let Proj ${ }_{C}$ be the metric projection from Hilbert space $\mathrm{H}_{1}$ onto $\mathrm{C}$ and let Proj $_{\mathrm{Q}}$ be the metric projection from Hilbert space $\mathrm{H}_{2}$ onto $\mathrm{Q}$. Let $\mathrm{S}: \mathrm{C} \rightarrow \mathrm{C}$ be a nonexpansive mapping with fixed 
points. Let $\mathrm{A}: \mathrm{H}_{1} \rightarrow \mathrm{H}_{2}$ be a bounded linear operator such that split feasibility problem (1.1) is consistent. Let $\left\{x_{n}\right\}$ be a sequence generated in the following iterative algorithm: $x_{1} \in \mathrm{C}$ is the initial and

$$
\left\{\begin{array}{l}
\lambda_{n}=\operatorname{Proj}_{C}\left(\left(1-\beta_{n}\right)\left(x_{n}-\gamma_{n} A^{*}\left(I-\operatorname{Proj}_{Q}\right) A x_{n}\right)+\beta_{n} S x_{n}\right), \\
x_{n+1}=\alpha_{n} u+\left(1-\alpha_{n}\right) \lambda_{n}, \quad n \geqslant 1,
\end{array}\right.
$$

where $u$ is a fixed element in $C,\left\{\alpha_{n}\right\},\left\{\beta_{n}\right\}$ are two sequences in $(0,1)$ with $\lim _{n \rightarrow \infty} \alpha_{n}=0, \sum_{n=1}^{\infty} \alpha_{n}=\infty$, $\sum_{n=1}^{\infty}\left|\alpha_{n}-\alpha_{n+1}\right|<\infty, 0<\liminf _{n \rightarrow \infty} \beta_{n} \leqslant \limsup _{n \rightarrow \infty} \beta_{n}<1, \sum_{n=1}^{\infty}\left|\beta_{n}-\beta_{n+1}\right|<\infty$, and $\left\{\gamma_{n}\right\}$ is a sequence with $0<\liminf _{n \rightarrow \infty} \gamma_{n} \leqslant \limsup _{n \rightarrow \infty} \gamma_{n}<\frac{2}{\|A\|^{2}}$ and $\sum_{n=1}^{\infty}\left|\gamma_{n}-\gamma_{n+1}\right|<\infty$. If Sol(SFP) $\cap$ $\operatorname{Fix}(S) \neq \emptyset$, then $\left\{x_{n}\right\}$ converges strongly to a point $\bar{u}$ in $\operatorname{Fix}(S) \cap \operatorname{Sol}(S F P)$, where $\bar{u}=\operatorname{Proj}_{\operatorname{Sol}(S F P) \cap F i x(S)} u$.

Proof. First, we show that sequence $\left\{x_{n}\right\}$ is bounded. Define a mapping $T: \mathrm{H}_{1} \rightarrow \mathrm{H}_{1}$ by

$$
\mathrm{T} x=A^{*}\left(\mathrm{I}-\operatorname{Proj}_{\mathrm{Q}}\right) A x, \quad \forall x \in \mathrm{H}_{1} .
$$

Using the properties of metric projection $\operatorname{Proj}_{\mathrm{Q}^{\prime}}$, we find that

$$
\begin{aligned}
\langle T x-T y, x-y\rangle & =\left\langle A^{*}\left(I-\operatorname{Proj}_{Q}\right) A x-A^{*}\left(I-\operatorname{Proj}_{Q}\right) A y, x-y\right\rangle \\
& =\left\langle\left(I-\operatorname{Proj}_{Q}\right) A x-\left(I-\operatorname{Proj}_{Q}\right) A y, A x-A y\right\rangle \\
& \geqslant\left\|\left(I-\operatorname{Proj}_{Q}\right) A x-\left(I-\operatorname{Proj}_{Q}\right) A y\right\|^{2} \\
& \geqslant \frac{1}{\|A\|^{2}}\left\|A^{*}\left(I-\operatorname{Proj}_{Q}\right) A x-A^{*}\left(I-\operatorname{Proj}_{Q}\right) A y\right\|^{2} \\
& =\frac{1}{\|A\|^{2}}\|T x-T y\|^{2} .
\end{aligned}
$$

This shows that $T$ is $\frac{1}{\|A\|^{2}}$-inverse-strongly monotone. From the restriction imposed on $\left\{\gamma_{n}\right\}$, we may, without loss of generality, assume that $0<\gamma \leqslant \gamma_{\mathrm{n}} \leqslant \bar{\gamma}<\frac{2}{\|A\|^{2}}$, where $\gamma$ and $\bar{\gamma}$ are real constants. Since $T$ is $\frac{1}{\|\mathrm{~A}\|^{2}}$-inverse-strongly monotone, we find that

$$
\begin{aligned}
\left\|\left(I-\gamma_{n} T\right) x-\left(I-\gamma_{n} T\right) y\right\|^{2} & =\left\|(x-y)-\gamma_{n}(T x-T y)\right\|^{2} \\
& =\gamma_{n}^{2}\|T x-T y\|^{2}+\|x-y\|^{2}-2 \gamma_{n}\langle x-y, T x-T y\rangle \\
& \leqslant \gamma_{n}^{2}\|T x-T y\|^{2}+\|x-y\|^{2}-\frac{2 \gamma_{n}}{\|A\|^{2}}\|T x-T y\|^{2} \\
& =\|x-y\|^{2}-\gamma_{n}\left(\frac{2}{\|A\|^{2}}-\gamma_{n}\right)\|T x-T y\|^{2} \\
& \leqslant\|x-y\|^{2} .
\end{aligned}
$$

This shows that $\left(I-\gamma_{n} T\right)$ is a nonexpansive mapping. Letting $x \in A^{-1}(Q)$, we find from the definition of $T$ that $x \in T^{-1}(0)$. This proves $A^{-1}(Q)$ is a subset of $T^{-1}(0)$. Letting $x \in T^{-1}(0)$, we have $T x=0$. Take a point $y \in \operatorname{Sol}(S F P) \cap \operatorname{Fix}(S)$. This implies $A y=\operatorname{Proj}_{Q} A y$. Hence, $T y=0$. Thanks to (2.1), one arrives at

$$
0=\langle T x-T y, x-y\rangle=\left\langle\left(I-\operatorname{Proj}_{Q}\right) A x-\left(I-\operatorname{Proj}_{Q}\right) A y, A x-A y\right\rangle \geqslant\left\|\left(I-\operatorname{Proj}_{Q}\right) A x\right\|^{2} .
$$

This proves $x \in A^{-1}(Q)$, that is, $T^{-1}(0)$ is a subset of $A^{-1}(Q)$. This completes the proof that

$$
\mathrm{T}^{-1}(0)=\mathrm{A}^{-1}(\mathrm{Q})
$$

It follows that

$$
\operatorname{Fix}(S) \cap \operatorname{Sol}(S F P)=\operatorname{Fix}(S) \cap T^{-1}(0) \cap C \text {. }
$$


On the other hand, we have

$$
\begin{aligned}
\left\|x_{n+1}-x^{*}\right\| & \leqslant\left(1-\alpha_{n}\right)\left\|\operatorname{Proj}_{C}\left(\left(1-\beta_{n}\right)\left(I-\gamma_{n} T\right) x_{n}+\beta_{n} S x_{n}\right)-\operatorname{Proj}_{C} x^{*}\right\|+\alpha_{n}\left\|u-x^{*}\right\| \\
& \leqslant\left(1-\alpha_{n}\right)\left\|\left(\left(1-\beta_{n}\right)\left(I-\gamma_{n} T\right) x_{n}+\beta_{n} S x_{n}\right)-x^{*}\right\|+\alpha_{n}\left\|u-x^{*}\right\| \\
& \leqslant\left(1-\alpha_{n}\right)\left(1-\beta_{n}\right)\left\|\left(I-\gamma_{n} T\right) x_{n}-x^{*}\right\|+\left(1-\alpha_{n}\right) \beta_{n}\left\|S x_{n}-x^{*}\right\|+\alpha_{n}\left\|u-x^{*}\right\| \\
& \leqslant\left(1-\alpha_{n}\right)\left\|x_{n}-x^{*}\right\|+\alpha_{n}\left\|u-x^{*}\right\| \\
& \leqslant \max _{\{}\left\{x_{n}-x^{*}\|,\| u-x^{*} \|\right\} .
\end{aligned}
$$

This proves that

$$
\left\|x_{n+1}-x^{*}\right\| \leqslant \max \left\{\left\|x_{1}-x^{*}\right\|,\left\|u-x^{*}\right\|\right\} .
$$

This completes the proof that $\left\{x_{n}\right\}$ is a bounded sequence. Note that

$$
\lambda_{n}=\operatorname{Proj}_{C}\left(\left(1-\beta_{n}\right)\left(I-\gamma_{n} T\right) x_{n}+\beta_{n} S x_{n}\right) .
$$

It follows that

$$
\begin{aligned}
\left\|\lambda_{n+1}-\lambda_{n}\right\| \leqslant & \left\|\left(\left(1-\beta_{n+1}\right)\left(I-\gamma_{n+1} T\right) x_{n+1}+\beta_{n+1} S x_{n+1}\right)-\left(\left(1-\beta_{n}\right)\left(I-\gamma_{n} T\right) x_{n}+\beta_{n} S x_{n}\right)\right\| \\
\leqslant & \left(1-\beta_{n+1}\right)\left\|\left(\left(I-\gamma_{n+1} T\right) x_{n+1}-\left(I-\gamma_{n} T\right) x_{n}\right)\right\| \\
& +\beta_{n+1}\left\|S x_{n+1}-S x_{n}\right\|+\left|\beta_{n+1}-\beta_{n}\right|\left\|\left(I-\gamma_{n} T\right) x_{n}-S x_{n}\right\| \\
\leqslant & \left(1-\beta_{n+1}\right)\left(\left\|x_{n+1}-x_{n}\right\|+\left|\gamma_{n}-\gamma_{n+1}\right|\left\|T x_{n}\right\|\right) \\
& +\beta_{n+1}\left\|x_{n+1}-x_{n}\right\|+\left|\beta_{n+1}-\beta_{n}\right|\left\|\left(I-\gamma_{n} T\right) x_{n}-S x_{n}\right\| \\
\leqslant & \left\|x_{n+1}-x_{n}\right\|+\left|\gamma_{n}-\gamma_{n+1}\right|\left\|T x_{n}\right\|+\left|\beta_{n+1}-\beta_{n}\right|\left\|\left(I-\gamma_{n} T\right) x_{n}-S x_{n}\right\| .
\end{aligned}
$$

This implies that

$$
\begin{aligned}
\left\|x_{n+2}-x_{n+1}\right\| \leqslant & \left|\alpha_{n+1}-\alpha_{n}\right|\left\|u-\lambda_{n}\right\|+\left(1-\alpha_{n+1}\right)\left\|\lambda_{n+1}-\lambda_{n}\right\| \\
\leqslant & \left\|u-\lambda_{n}\right\|+\left(1-\alpha_{n+1}\right)\left\|x_{n+1}-x_{n}\right\| \\
& +\left|\gamma_{n}-\gamma_{n+1}\right|\left\|T x_{n}\right\|+\left|\beta_{n+1}-\beta_{n}\right|\left\|\left(I-\gamma_{n} T\right) x_{n}-S x_{n}\right\| \\
\leqslant & \left(1-\alpha_{n+1}\right)\left\|x_{n+1}-x_{n}\right\|+\left(\left|\gamma_{n}-\gamma_{n+1}\right|+\left|\beta_{n+1}-\beta_{n}\right|+\left|\alpha_{n+1}-\alpha_{n}\right|\right) M,
\end{aligned}
$$

where

$$
M=\max \left\{\sup _{n \geqslant 1}\left\{\left\|u-\lambda_{n}\right\|\right\}, \sup _{n \geqslant 1}\left\{\left\|T x_{n}\right\|\right\}, \sup _{n \geqslant 1}\left\{\left\|\left(I-\gamma_{n} T\right) x_{n}-S x_{n}\right\|\right\}\right\} .
$$

Using Lemma 1.1, we find that

$$
\lim _{n \rightarrow \infty}\left\|x_{n+1}-x_{n}\right\|=0
$$

On the other hand, we have

$$
\begin{aligned}
\left\|x^{*}-\left(I-\gamma_{n} T\right) x_{n}\right\|^{2} & =\left\|\left(x_{n}-x^{*}\right)-\gamma_{n}\left(T x_{n}-T x^{*}\right)\right\|^{2} \\
& \leqslant \gamma_{n}^{2}\left\|T x_{n}-T x^{*}\right\|^{2}+\left\|x_{n}-x^{*}\right\|^{2}-\frac{2 \gamma_{n}}{\|A\|^{2}}\left\|T x_{n}-T x^{*}\right\|^{2} \\
& =\left\|x_{n}-x^{*}\right\|^{2}-\left(\frac{2 \gamma_{n}}{\|A\|^{2}}-\gamma_{n}^{2}\right)\left\|T x_{n}-T x^{*}\right\|^{2} \\
& =\left\|x_{n}-x^{*}\right\|^{2}-\left(\frac{2 \gamma_{n}}{\|A\|^{2}}-\gamma_{n}^{2}\right)\left\|T x_{n}\right\|^{2} .
\end{aligned}
$$

This in turn implies from (2.3) that

$$
\begin{aligned}
\left\|\lambda_{n}-x^{*}\right\|^{2} & \leqslant\left\|\left(\left(1-\beta_{n}\right)\left(I-\gamma_{n} T\right) x_{n}+\beta_{n} S x_{n}\right)-x^{*}\right\| \\
& \leqslant\left(1-\beta_{n}\right)\left\|x^{*}-\left(I-\gamma_{n} T\right) x_{n}\right\|+\beta_{n}\left\|S x_{n}-x^{*}\right\| \\
& \leqslant\left\|x^{*}-x_{n}\right\|^{2}-\left(1-\beta_{n}\right)\left(\frac{2 \gamma_{n}}{\|A\|^{2}}-\gamma_{n}^{2}\right)\left\|T x_{n}\right\|^{2} .
\end{aligned}
$$


Hence, we have

$$
\begin{aligned}
\left\|x_{n+1}-x^{*}\right\|^{2} & \leqslant \alpha_{n}\left\|u-x^{*}\right\|^{2}+\left(1-\alpha_{n}\right)\left\|\lambda_{n}-x^{*}\right\|^{2} \\
& \leqslant \alpha_{n}\left\|u-x^{*}\right\|^{2}+\left(1-\alpha_{n}\right)\left\|x^{*}-x_{n}\right\|^{2}-\left(1-\alpha_{n}\right)\left(1-\beta_{n}\right)\left(\frac{2 \gamma_{n}}{\|A\|^{2}}-\gamma_{n}^{2}\right)\left\|T x_{n}\right\|^{2} \\
& \leqslant \alpha_{n}\left\|u-x^{*}\right\|^{2}+\left\|x^{*}-x_{n}\right\|^{2}-\left(1-\alpha_{n}\right)\left(1-\beta_{n}\right)\left(\frac{2 \gamma_{n}}{\|A\|^{2}}-\gamma_{n}^{2}\right)\left\|T x_{n}\right\|^{2} .
\end{aligned}
$$

It follows that

$$
\begin{aligned}
\left(1-\alpha_{n}\right)\left(1-\beta_{n}\right)\left(\frac{2 \gamma_{n}}{\|A\|^{2}}-\gamma_{n}^{2}\right)\left\|T x_{n}\right\|^{2} & \leqslant \alpha_{n}\left\|u-x^{*}\right\|^{2}+\left\|x_{n}-p\right\|^{2}-\left\|x_{n+1}-p\right\|^{2} \\
& \leqslant \alpha_{n}\left\|u-x^{*}\right\|^{2}+\left(\left\|x_{n}-p\right\|+\left\|x_{n+1}-p\right\|\right)\left\|x_{n+1}-x_{n}\right\| .
\end{aligned}
$$

From the restriction imposed on $\left\{\beta_{n}\right\}$, we may, without loss of generality, assume that $0<\beta \leqslant \beta_{n} \leqslant \bar{\beta}<$ $\frac{2}{\|A\|^{2}}$, where $\beta$ and $\bar{\beta}$ are real constants. We find from (2.2) that

$$
\lim _{n \rightarrow \infty}\left\|T x_{n}\right\|=0 \text {. }
$$

Set $\bar{u}=\operatorname{Proj}_{\operatorname{Sol}(S F P) \cap F i x(S)} u$. Now, we are in a position to show that

$$
\limsup _{n \rightarrow \infty}\left\langle u-\bar{u}, \lambda_{n}-\bar{u}\right\rangle \leqslant 0
$$

To this end, we take a subsequence $\left\{x_{n_{j}}\right\}$ of $\left\{x_{n}\right\}$ such that

$$
\limsup _{n \rightarrow \infty}\left\langle u-\bar{u}, x_{n}-\bar{u}\right\rangle=\lim _{j \rightarrow \infty}\left\langle u-\bar{u}, x_{n_{j}}-\bar{u}\right\rangle .
$$

Without loss of generality, let us assume that $\left\{x_{n_{j}}\right\}$ converges weakly to a point in C. Next we denote the point by $\omega$. Setting $W=I-T$, we see that $W$ is nonexpansive. From the demiclosed principal of $W$, we find from (2.4) that

$$
\omega \in \operatorname{Fix}(W)=T^{-1}(0)=A^{-1}(Q) .
$$

On the other hand, we find that

$$
\begin{aligned}
\left\|x_{n}-S x_{n}\right\| & \leqslant\left\|x_{n}-x_{n+1}\right\|+\alpha_{n}\left\|u-S x_{n}\right\|+\left(1-\alpha_{n}\right)\left\|\lambda_{n}-S x_{n}\right\| \\
& \leqslant\left\|x_{n}-x_{n+1}\right\|+\alpha_{n}\left\|u-S x_{n}\right\|+\left(1-\alpha_{n}\right)\left(1-\beta_{n}\right)\left\|\left(x_{n}-T\right) x_{n}-S x_{n}\right\| \\
& \leqslant\left\|x_{n}-x_{n+1}\right\|+\alpha_{n}\left\|u-S x_{n}\right\|+\left(1-\beta_{n}\right)\left\|x_{n}-S x_{n}\right\|+\left\|T x_{n}\right\| .
\end{aligned}
$$

This finds that

$$
\left\|x_{n}-S x_{n}\right\| \leqslant \frac{1}{\beta_{n}}\left\|x_{n}-x_{n+1}\right\|+\frac{\alpha_{n}}{\beta_{n}}\left\|u-S x_{n}\right\|+\frac{1}{\beta_{n}}\left\|T x_{n}\right\| .
$$

Using (2.2) and (2.4), we find that

$$
\lim _{n \rightarrow \infty}\left\|x_{n}-S x_{n}\right\|=0 .
$$

From the demiclosed principal of $S$, we find that $\omega \in \operatorname{Fix}(S)$. This obtains that (2.5) holds.

Note that

$$
\begin{aligned}
\left\|x_{n+1}-\bar{u}\right\|^{2} \leqslant & \alpha_{n}^{2}\|u-\bar{u}\|^{2}+\left(1-\alpha_{n}\right)^{2}\left\|\lambda_{n}-\bar{u}\right\|^{2}+2 \alpha_{n}\left(1-\alpha_{n}\right)\left\langle u-\bar{u}, \lambda_{n}-\bar{u}\right\rangle \\
\leqslant & \alpha_{n}^{2}\|u-\bar{u}\|^{2}+\left(1-\alpha_{n}\right)^{2}\left(\left(1-\beta_{n}\right)\left\|\left(I-\gamma_{n} T\right) x_{n}-\bar{u}\right\|+\beta_{n}\left\|S x_{n}-\bar{u}\right\|\right)^{2} \\
& +2 \alpha_{n}\left(1-\alpha_{n}\right)\left\langle u-\bar{u}, \lambda_{n}-\bar{u}\right\rangle \\
\leqslant & \left(1-\alpha_{n}\right)^{2}\left(1-\beta_{n}\right)^{2}\left\|x_{n}-\bar{u}\right\|^{2}+\alpha_{n}^{2}\|u-\bar{u}\|^{2}+2 \alpha_{n}\left(1-\alpha_{n}\right)\left\langle u-\bar{u}, \lambda_{n}-\bar{u}\right\rangle \\
\leqslant & \left(1-\alpha_{n}\right)\left\|x_{n}-\bar{u}\right\|^{2}+\alpha_{n} \xi_{n},
\end{aligned}
$$

where

$$
\xi_{n}=\alpha_{n}\|u-\bar{u}\|^{2}+2\left\langle u-\bar{u}, \lambda_{n}-\bar{u}\right\rangle .
$$

Using Lemma 1.1, we find that $x_{n} \rightarrow \bar{u}$ as $n \rightarrow \infty$. This completes the proof. 
If $S=I$, the identity mapping, then Theorem 2.1 is reduced to the following result.

Corollary 2.2. Let $\mathrm{C}$ be a nonempty closed and convex subset of Hilbert space $\mathrm{H}_{1}$ and let $\mathrm{Q}$ be a nonempty closed and convex subset of Hilbert space $\mathrm{H}_{2}$. Let Proj ${ }_{C}$ be the metric projection from Hilbert space $\mathrm{H}_{1}$ onto $\mathrm{C}$ and let Proj $_{\mathrm{Q}}$ be the metric projection from Hilbert space $\mathrm{H}_{2}$ onto $\mathrm{Q}$. Let $\mathrm{S}: \mathrm{C} \rightarrow \mathrm{C}$ be a nonexpansive mapping with fixed points. Let $\mathrm{A}: \mathrm{H}_{1} \rightarrow \mathrm{H}_{2}$ be a bounded linear operator such that split feasibility problem (1.1) is consistent. Let $\left\{x_{n}\right\}$ be a sequence generated in the following iterative algorithm: $x_{1} \in \mathrm{C}$ is the initial and

$$
\left\{\begin{array}{l}
\lambda_{n}=\operatorname{Proj}_{C}\left(x_{n}-\left(1-\beta_{n}\right) \gamma_{n} A^{*}\left(I-\operatorname{Proj}_{Q}\right) A x_{n}\right), \\
x_{n+1}=\alpha_{n} u+\left(1-\alpha_{n}\right) \lambda_{n}, \quad n \geqslant 1,
\end{array}\right.
$$

where $u$ is a fixed element in $C,\left\{\alpha_{n}\right\}$ and $\left\{\beta_{n}\right\}$ are two sequences in $(0,1)$ with $\lim _{n \rightarrow \infty} \alpha_{n}=0, \sum_{n=1}^{\infty} \alpha_{n}=\infty$, $\sum_{n=1}^{\infty}\left|\alpha_{n}-\alpha_{n+1}\right|<\infty, 0<\liminf _{n \rightarrow \infty} \beta_{n} \leqslant \limsup _{n \rightarrow \infty} \beta_{n}<1, \sum_{n=1}^{\infty}\left|\beta_{n}-\beta_{n+1}\right|<\infty$, and $\left\{\gamma_{n}\right\}$ is a sequence with $0<\liminf _{n \rightarrow \infty} \gamma_{n} \leqslant \limsup _{n \rightarrow \infty} \gamma_{n}<\frac{2}{\|A\|^{2}}$ and $\sum_{n=1}^{\infty}\left|\gamma_{n}-\gamma_{n+1}\right|<\infty$. Then $\left\{x_{n}\right\}$ converges strongly to a point $\overline{\mathrm{u}}$ in $\operatorname{Sol}(\mathrm{SFP})$, where $\overline{\mathrm{u}}=\operatorname{Proj}_{\mathrm{Sol}(\mathrm{SFP})} \mathrm{u}$.

Remark 2.3. Fixed point methods are investigated to split feasibility problem (1.1). A strong convergence theorem of common solutions are established in the framework of infinite dimensional Hilbert spaces without any compact assumption. It is of interest to improve the results presented in this article to the framework of Banach spaces.

\section{Acknowledgment}

The authors are grateful to the referees for useful suggestions which improved the contents of this article.

\section{References}

[1] B. A. Bin Dehaish, A. Latif, H. O. Bakodah, X. Qin, A regularization projection algorithm for various problems with nonlinear mappings in Hilbert spaces, J. Inequal. Appl., 2015 (2015), 14 pages. 1

[2] B. A. Bin Dehaish, X. Qin, A. Latif, H. Bakodah, Weak and strong convergence of algorithms for the sum of two accretive operators with applications, J. Nonlinear Convex Anal., 16 (2015), 1321-1336. 1

[3] F. E. Browder, Fixed-point theorems for noncompact mappings in Hilbert space, Proc. Natl. Acad. Sci. U.S.A., 53 (1965), 1272-1276. 1, 1.2

[4] C. Byrne, A unified treatment of some iterative algorithms in signal processing and image reconstruction, Inverse Probl., 20 (2004), 103-120. 1

[5] Y. Censor, T. Bortfeld, B. Martin, A. Trofimov, A unified approach for inversion problems in intensity modulated radiation therapy, Phys. Med. Biol., 51 (2006), 2353-2365. 1

[6] Y. Censor, T. Elfving, A multiprojection algorithm using Bregman projections in a product space, Numer. Algorithms, 8 (1994), 221-239. 1

[7] Y. Censor, T. Elfving, N. Kopf, T. Bortfeld, The multiple-sets split feasibility problem and its applications for inverse problems, Inverse Probl., 21 (2005), 2071-2084. 1

[8] S. Y. Cho, B. A. Bin Dehaish, X. Qin, Weak convergence of a splitting algorithm in Hilbert spaces, J. Appl. Anal. Comput., 7 (2017), 427-438. 1

[9] S. Y. Cho, S. M. Kang, Approximation of common solutions of variational inequalities via strict pseudocontractions, Acta Math. Sci. Ser. B Engl. Ed., 32 (2012), 1607-1618. 1

[10] S. Y. Cho, W. Li, S. M. Kang, Convergence analysis of an iterative algorithm for monotone operators, J. Inequal. Appl., 2013 (2013), 14 pages. 1

[11] N. Fang, Y. Gong, Viscosity iterative methods for split variational inclusion problems and fixed poit problems of a nonexpansive mappings, Commun. Optim. Theory, 2016 (2016), 15 pages. 1

[12] L. S. Liu, Ishikawa and Mann iterative process with errors for nonlinear strongly accretive mappings in Banach spaces, J. Math. Anal. Appl., 194 (1995), 114-125. 1.1

[13] X. Qin, S. S. Chang, Y. J. Cho, Iterative methods for generalized equilibrium problems and fixed point problems with applications, Nonlinear Anal. Real World App., 11 (2010), 2963-2972. 1

[14] X. Qin, S. Y. Cho, Convergence analysis of a monotone projection algorithm in reflexive banach spaces, Acta Math. Sci. Ser. B Engl. Ed., 37 (2017), 488-502. 1 
[15] X. Qin, J. C. Yao, Weak convergence of a Mann-like algorithm for nonexpansive and accretive operators, J. Inequal. Appl., 2016 (2016), 9 pages. 1

[16] W. Takahahsi, Weak and strong convergence theorems for families of nonlinear and nonself mappings in Hilbert spaces, J. Nonlinear Var. Anal., 1 (2017), 1-23. 1

[17] J. Tang, S. S. Chang, Strong convergence theorem of two-step iterative algorithm for split feasibility problems, J. Inequal. Appl., 2014 (2014), 13 pages. 1

[18] J. Tang, S. S. Chang, J. Dong, Split equality fixed point problems for two quasi-asymptotically pseudocontractive mappings, J. Nonlinear Funct. Anal., 2017 (2017), 15 pages.

[19] H. Y. Zhou, Y. Wang, Adaptively relaxed algorithms for solving the split feasibility problem with a new step size, J. Inequal. Appl., 2014 (2014), 22 pages. 1 